\title{
Quasi-one-dimensional Bose-Einstein condensates in nonlinear lattices
}

\author{
L. Salasnich ${ }^{1}$ and B.A. Malomed ${ }^{2}$ \\ ${ }^{1}$ Dipartimento di Fisica "Galileo Galilei" and CNISM, Università di Padova, Via \\ Marzolo 8, 35131 Padova, Italy \\ ${ }^{2}$ Department of Physical Electronics, School of Electrical Engineering, Faculty of \\ Engineering, Tel Aviv University, Tel Aviv 69978, Israel \\ E-mail: luca.salasnich@unipd.it
}

\begin{abstract}
We consider the three-dimensional (3D) mean-field model for the BoseEinstein condensate (BEC), with a 1D nonlinear lattice (NL), which periodically changes the sign of the nonlinearity along the axial direction, and the harmonicoscillator trapping potential applied in the transverse plane. The lattice can be created as an optical or magnetic one, by means of available experimental techniques. The objective is to identify stable 3D solitons supported by the setting. Two methods are developed for this purpose: The variational approximation, formulated in the framework of the 3D Gross-Pitaevskii equation, and the 1D nonpolynomial Schrödinger equation (NPSE) in the axial direction, which allows one to predict the collapse in the framework of the 1D description. Results are summarized in the form of a stability region for the solitons in the plane of the NL strength and wavenumber. Both methods produce a similar form of the stability region. Unlike their counterparts supported by the NL in the 1D model with the cubic nonlinearity, kicked solitons of the NPSE cannot be set in motion, but the kick may help to stabilize them against the collapse, by causing the solitons to shed excess norm. A dynamical effect specific to the NL is found in the form of freely propagating small-amplitude wave packets emitted by perturbed solitons.
\end{abstract}




\section{Introduction and the model}

The use of periodic potentials, induced by optical lattices, for steering matter waves in Bose-Einstein condensates (BECs) is a vast research area, as demonstrated in reviews [1][4]. An important aspect of this topic is that the lattice potentials, balancing the cubic nonlinearities induced by inter-atomic collisions in the BEC, help to create and stabilize solitons. In particular, the lattices play a critical role in stabilizing two-dimensional (2D) solitons against the collapse in the condensate with intrinsic self-attraction [5].

Theoretical and experimental studies of the soliton dynamics in periodic potentials were recently extended for nonlinear lattices (NLs), which may be induced by a spatially periodic modulation of the local strength of the nonlinearity (the respective effective nonlinear potentials are often called pseudopotentials [6]). In BEC, the spatial modulation can be implemented by means of the Feshbach resonance controlled by properly patterned external fields (in the quasi-1D BEC, a combination of linear and nonlinear lattices may also be induced by periodically modulating the strength of the tight transverse confinement in the axial direction [7]). As well as their linear counterparts, NLs have drawn much attention in connection to their potential for the creation and control of matter-wave solitons in a number of different settings, see original works [8]-[18] and review [4]. While NLs readily support stable 1D solitons [8]-[17], it has been found difficult, albeit sometimes possible, to stabilize 2D solitons against the collapse by means of the NL-induced pseudopotentials [18]. As concerns the 1D solitons in models with the cubic nonlinearity, the numerical analysis also reveals that they feature mobility in the presence of NLs [8, 9].

Thus far, no example of stabilization of 3D solitons by NLs has been reported. On the other hand, the creation of multidimensional solitons can be facilitated by combinations of linear and nonlinear lattices, as shown, in particular, in Ref. [11, where 2D solitons supported by crossed 1D lattices, one linear and one nonlinear, were reported. Still earlier, it was demonstrated that the $1 \mathrm{D}$ linear-lattice potential, acting together with periodic temporal modulations of the nonlinearity, which may be induced by the Feshbach resonance controlled by a time-periodic external field, can stabilize 3D solitons [19].

A natural possibility for the creation of 3D solitons is suggested by a combination of the 1D NL with the usual harmonic-oscillator linear trapping potential acting in the plane perpendicular to the NL axis (similar settings, but with linear 1D lattices, were shown to be efficient in the creation of 3D gap solitons in the BEC with the repulsive intrinsic nonlinearity [20]). This setting is the subject of the present paper. We tackle it by means of two different methods, namely, the variational approximation (VA) applied to the underlying 3D Gross-Pitaevskii equation, and, in a more accurate form, the effective 1D nonpolynomial Schrödinger equation (NPSE), which was efficient in description of many other settings dominated by the interplay of the tight 2D confinement and nonlinearity [21, [22, [7], including the action of linear lattice potentials in the axial direction [23]. 
Thus, we consider a dilute BEC of bosons with mass $m$ confined in the transverse plane by the isotropic harmonic-oscillator potential with frequency $\omega_{\perp}, V(x, y)=$ $(1 / 2) m \omega_{\perp}^{2}\left(x^{2}+y^{2}\right)$. The corresponding 3D Gross-Pitaevskii equation is rescaled by measuring time and coordinates in units of $\omega_{\perp}^{-1}$ and the transverse-confinement radius, $a_{\perp}=\sqrt{\hbar /\left(m \omega_{\perp}\right)}$, respectively (hence the energy is measured in units of $\hbar \omega_{\perp}$ ):

$$
i \frac{\partial \psi}{\partial t}=\left[-\frac{1}{2} \nabla^{2}+\frac{1}{2}\left(x^{2}+y^{2}\right)+2 \pi g(z)|\psi|^{2}\right] \psi,
$$

with the condensate's wave function normalized to unity,

$$
\int|\psi(\mathbf{r}, t)|^{2} d^{3} \mathbf{r}=1
$$

The interaction strength in Eq. (11) is

$$
g(z)=2(N-1) a_{s}(z) / a_{\perp},
$$

where $N$ is the number of atoms and $a_{s}$ the $z$-dependent $s$-wave scattering length of the inter-atomic potential, the NL corresponding to the periodic dependence,

$$
g(z)=g_{0}+g_{1} \cos (2 k z) .
$$

Below, we consider the most fundamental case of $g_{0}=0$. Placing the center of the soliton at $z=0$, we assume $g_{1}<0$, to support the soliton by the locally attractive nonlinearity.

The 1D periodic modulation of the local scattering length, implied by Eqs. (4) and (3), can be implemented in an optical lattice, produced by the interference of a pair of counterpropagating laser beams controlling $a_{s}$ via the Feshbach resonance [24]. In that case, the period of the resulting NL is limited by diffraction to $\gtrsim 1 \mu \mathrm{m}$. More often, the Feshbach resonance in experiments with BEC is controlled by the magnetic field [25]. In that case, the 1D periodic structure can be built as a magnetic lattice, imposed by a properly designed set of ferromagnet films [26], with the respective fabrication limit on the NL period also amounting to $\gtrsim 1 \mu \mathrm{m}$. Then, assuming that the trapping potential confines the transverse size of the condensate, as usual, to the same order of magnitude (a few microns), one may conclude that the solitons are built of several thousand atoms [27].

The rest of the paper is organized as follows. The direct VA is developed in Section II, and the effective 1D NPSE is derived in Section III. In both cases, we find the stability region for solitons in the plane of $\left(k,\left|g_{1}\right|\right)$. The mobility of the solitons is tested in Section III by means of direct simulations of the evolution of axially kicked solitons, in the framework of the NPSE (it is concluded that the solitons are not mobile in the present setting; however, the kick may effectively stabilize solitons against the collapse). The paper is concluded by Section IV. 


\section{The variational approximation}

Our first aim is to apply the VA to Eq. (11), following the lines of Ref. [31]. To this end, we notice that Eq. (1) can be derived from the Lagrangian density,

$$
\mathcal{L}=\frac{i}{2}\left(\psi^{*} \frac{\partial \psi}{\partial t}-\psi \frac{\partial \psi^{*}}{\partial t}\right)-\frac{1}{2}|\nabla \psi|^{2}-\frac{1}{2}\left(x^{2}+y^{2}\right)|\psi|^{2}-\pi g(z)|\psi|^{4},
$$

and make use of a time-dependent Gaussian ansatz,

$$
\psi(\mathbf{r}, t)=\frac{\exp \left\{-\frac{1}{2}\left[\frac{r_{\perp}^{2}}{\sigma_{\perp}^{2}(t)}+\frac{z^{2}}{\sigma_{\|}^{2}(t)}\right]+i \beta_{\perp}(t) r_{\perp}^{2}+i \beta_{\|}(t) z^{2}\right\}}{\pi^{3 / 4} \sigma_{\perp}(t) \sqrt{\sigma_{\|}(t)}},
$$

where $r_{\perp}^{2} \equiv x^{2}+y^{2}$, and $\sigma_{\perp}(t), \sigma_{\|}(t)$ and $\beta_{\perp}(t), \beta_{\|}(t)$ are time-dependent variational parameters. This wave function is an exact one for non-interacting bosons $(g=0)$ in the harmonic trap.

Inserting the ansatz into Lagrangian density (5) and performing the spatial integration, we arrive at the effective Lagrangian,

$$
\begin{aligned}
L & =-\frac{1}{2}\left[\left(2 \dot{\beta}_{\perp} \sigma_{\perp}^{2}+\frac{1}{\sigma_{\perp}^{2}}+4 \sigma_{\perp}^{2} \beta_{\perp}^{2}+\sigma_{\perp}^{2}\right)\right. \\
& \left.+\left(\dot{\beta}_{\|} \sigma_{\|}^{2}+\frac{1}{2 \sigma_{\|}^{2}}+2 \sigma_{\|}^{2} \beta_{\|}^{2}\right)+\frac{g_{0}+g_{1} e^{-k^{2} \sigma_{\|}^{2} / 2}}{\sqrt{2 \pi} \sigma_{\perp}^{2} \sigma_{\|}}\right],
\end{aligned}
$$

with the overdot standing for time derivatives. The respective Euler-Lagrange equations take the form of

$$
\begin{aligned}
\beta_{\perp} & =-\frac{\dot{\sigma}_{\perp}}{2 \sigma_{\perp}}, \\
\beta_{\|} & =-\frac{\dot{\sigma}_{\|}}{2 \sigma_{\|}}, \\
\ddot{\sigma}_{\perp}+\sigma_{\perp} & =\frac{1}{\sigma_{\perp}^{3}}+\frac{g_{0}+g_{1} e^{-k^{2} \sigma_{\|}^{2} / 2}}{\sqrt{2 \pi} \sigma_{\perp}^{3} \sigma_{\|}}, \\
\ddot{\sigma}_{\|} & =\frac{1}{\sigma_{\|}^{3}}+\frac{g_{0}+g_{1} e^{-k^{2} \sigma_{\|}^{2} / 2}\left(1+k^{2} \sigma_{\|}^{2}\right)}{\sqrt{2 \pi} \sigma_{\perp}^{2} \sigma_{\|}^{2}} .
\end{aligned}
$$

Equations (9) and (10) show that, as usual, chirps $\beta_{\perp}$ and $\beta_{\|}$are determined by the time dependence of $\sigma_{\perp}$ and $\sigma_{\|}$, while Eqs. (11) and (12) correspond to the equations of motion of a mechanical system with two degrees of freedom, whose energy is

$$
\begin{aligned}
& E=\frac{1}{2} \dot{\sigma}_{\perp}^{2}+\frac{1}{4} \dot{\sigma}_{\|}^{3}+U\left(\sigma_{\perp}, \sigma_{\|}\right), \\
& U\left(\sigma_{\perp}, \sigma_{\|}\right)=\frac{1}{2} \sigma_{\perp}^{2}+\frac{1}{2 \sigma_{\perp}^{2}}+\frac{1}{4 \sigma_{\|}^{2}}+\frac{g_{0}+g_{1} e^{-k^{2} \sigma_{\|}^{2} / 2}}{2 \sqrt{2 \pi} \sigma_{\perp}^{2} \sigma_{\|}} .
\end{aligned}
$$

Next, we look for stationary configurations corresponding to minima of potential energy (13), by demanding $\partial U / \partial \sigma_{\perp}=\partial U / \partial \sigma_{\|}=0$, which yields

$$
\sigma_{\perp}=\frac{1}{\sigma_{\perp}^{3}}+\frac{g_{0}+g_{1} e^{-k^{2} \sigma_{\|}^{2} / 2}}{\sqrt{2 \pi} \sigma_{\perp}^{3} \sigma_{\|}}
$$




$$
0=\frac{1}{\sigma_{\|}^{3}}+\frac{g_{0}+g_{1} e^{-k^{2} \sigma_{\|}^{2} / 2}\left(1+k^{2} \sigma_{\|}^{2}\right)}{\sqrt{2 \pi} \sigma_{\perp}^{2} \sigma_{\|}^{2}} .
$$

These equations, which are fixed points of Eqs. (11) and (12), with $\ddot{\sigma}_{\perp}=\ddot{\sigma}_{\|}=0$, can be solved numerically. The solutions provide for a minimum of the energy under the necessary condition that the Gaussian curvature $K_{G}$ of energy surface $U\left(\sigma_{\perp}, \sigma_{\|}\right)$is positive, i.e.,

$$
K_{G} \equiv \frac{\partial^{2} U}{\partial \sigma_{\perp}^{2}} \frac{\partial^{2} U}{\partial \sigma_{\|}^{2}}-\left(\frac{\partial^{2} U}{\partial \sigma_{\perp} \partial \sigma_{\|}}\right)^{2}>0 .
$$

Further, low-energy excitations of the condensate around the stationary state are represented by small oscillations of variables $\sigma_{\perp}$ and $\sigma_{\|}$around the equilibrium point defined by Eqs. (14) and (15). The calculation of the corresponding normal-mode frequencies, $\Omega$, is thus reduced to finding eigenvalues of the respective Hessian matrix,

$$
\Lambda=\left(\begin{array}{cc}
\partial^{2} U / \partial \sigma_{\perp}^{2} & \partial^{2} U / \partial \sigma_{\perp} \partial \sigma_{\|} \\
\partial^{2} U / \partial \sigma_{\|} \partial \sigma_{\perp} & \partial^{2} U / \partial \sigma_{\|}^{2}
\end{array}\right),
$$

while the associated mass matrix is

$$
M=\left(\begin{array}{cc}
1 & 0 \\
0 & \frac{1}{2}
\end{array}\right) .
$$

Then, the eigenfrequencies are found as the solutions of equation

$$
\operatorname{det}\left(\Lambda-\Omega^{2} M\right)=0 \text {. }
$$

Widths $\sigma_{\perp}$ and $\sigma_{\|}$of stable solitons, as found from the numerical solution of Eqs. (14) and (15), are plotted in the left panels of Fig. 1 as functions of the NL strength parameter, $\left|g_{1}\right|$ [recall we set $g_{0}=0$ and $g_{1}<0$ in Eq. (44)], along with the widths obtained by solving numerically the 1D NPSE (see below). The comparison demonstrates close proximity of the predictions of the VA to the results produced by the NPSE. In the right panels of Fig. 1, we plot eigenfrequencies $\Omega_{1}$ and $\Omega_{2}$ of excitations around the bright soliton, as found from Eq. (18).

In Fig. 2 we plot the stability diagram in the plane of $\left(k,\left|g_{1}\right|\right)$ for the bright solitons trapped in the NL with wavenumber $k$ and strength $\left|g_{1}\right|$ (again, with $g_{0}=0$ ). The stability region, defined as that where solutions of Eqs. (14) and (15) yield energy minima, is bounded by the dashed lines (strictly speaking, the bright solitons are only metastable, because for $g_{0}=0$ and $g_{1}<0$ the true ground state is the collapsed one, with potential energy $U=-\infty)$. Below the lower dashed line, the soliton is subject to spreading along longitudinal axis $z$ (which may be considered as a manifestation of the delocalization transition, which was earlier studied in linear lattices [30]). Above the upper dashed line, the soliton is destroyed by the collapse. Very close to the upper dashed line, the VA predicts a bistability, i.e., coexistence of two stable solitons at the same values of $k$ and $\left|g_{1}\right|$ (in Fig. 1, only the solitons with the lowest energy are shown in the case of the bistability). In Fig. 2 we also plot the respective stability region (between the solid lines) as obtained from the numerical solution of the NPSE (see 

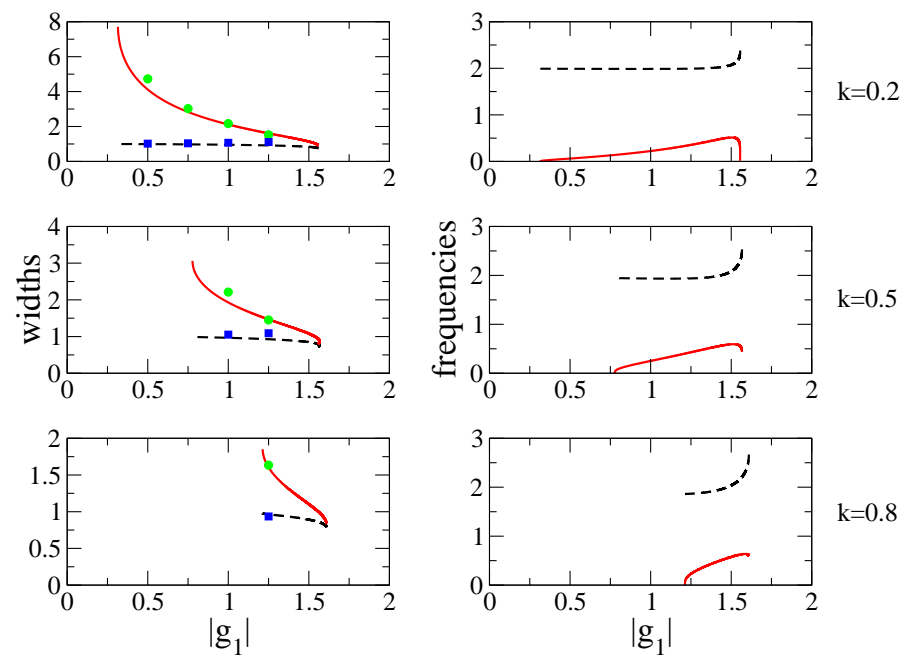

Figure 1. (Color online) Left panels: transverse and axial widths, $\sigma_{\perp}$ and $\sigma_{\|}$(dashed and solid lines, respectively) of stable bright solitons, as functions of the NL strength, $\left|g_{1}\right|$, with $g_{0}=0$ and $g_{1}<0$, obtained from the variational approximation. Filled circles and squares depict the widths obtained from a numerical solution of the NPSE. Right panels: two eigenfrequencies $\Omega_{1}$ and $\Omega_{2}$ (dashed and solid lines) of collective excitations around the stable bright soliton, vs. $\left|g_{1}\right|$, as obtained from the variational approximation. The results are shown for three values of the NL wavenumber: $k=0.2$ (upper panels), $k=0.5$ (middle panels), and $k=0.8$ (lower panels).

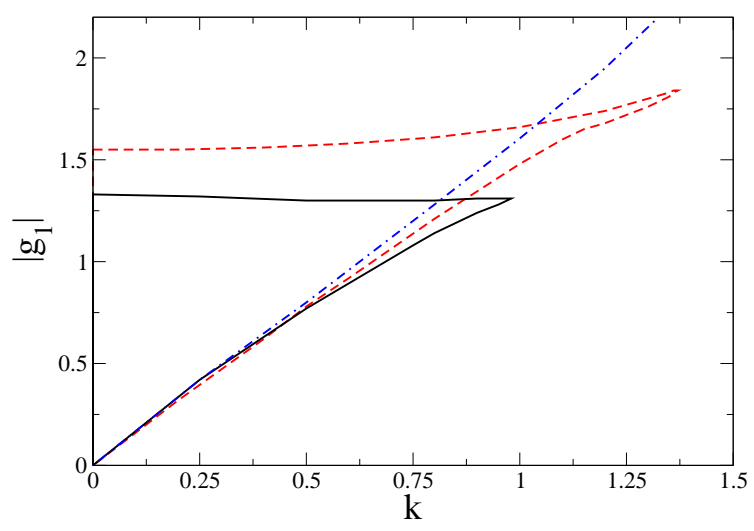

Figure 2. (Color online) The stability diagram for the solitons in the plane of wavenumber $k$ and strength $\left|g_{1}\right|$ of the NL (with $g_{0}=0$ ). The solitons are stable between the dashed lines, according to the variational approximation, and between the solid lines, according to the NPSE. The dot-dashed line is the lower bound predicted by the one-dimensional cubic Gross-Pitaevskii equation.

below). The shrinkage and disappearance of the stability region at large values of $k$ is quite natural, as in that case the rapidly oscillating nonlinearity in Eq. (4) tends to average itself to zero.

The prediction of the usual 1D cubic Gross-Pitaevskii equation is also plotted in Fig. 2. In that case, there is only one stability boundary (the dot-dashed line), as the 
1D equation with the cubic nonlinearity does not predict the collapse. Accordingly, the above argument concerning the disappearance of the stability region at large $k$ does not apply to the cubic equation, because, in the limit of the small NL period, $\pi / k$, the soliton may compress itself into a single potential well, and this trend will not be aborted by the onset of the collapse.

\section{The nonpolynomial Schrödinger equation (NPSE)}

\subsection{The derivation and imaginary-time evolution}

A more accurate description of the solitons is provided by the NPSE, which can be derived by means of the semi-variational approach from the full 3D equation (1), using the method developed in Ref. [21]. To this end, we adopt the following ansatz, which, unlike the above one (6), contains arbitrary functions of the longitudinal coordinate, $\sigma(z, t)$ and $f(z, t)$, accounting for the transverse width and axial wave function of the condensate:

$$
\psi(\mathbf{r}, t)=\frac{1}{\sqrt{\pi} \sigma(z, t)} \exp \left[-\frac{x^{2}+y^{2}}{2(\sigma(z, t))^{2}}\right] f(z, t) .
$$

Note that, as follows from Eqs. (19) and (2), the norm of the axial wave function is also 1:

$$
N_{1 \mathrm{D}} \equiv \int_{-\infty}^{+\infty}|f(z)|^{2} d z=1
$$

Substituting ansatz (19) into Lagrangian density (5), performing the integration over $x$ and $y$, and omitting spatial derivatives of the transverse width (this corresponds to the adiabatic approximation, which is known to produce accurate results in other settings [21]), we derive the respective Lagrangian density,

$$
\overline{\mathcal{L}}=\frac{i}{2}\left(f^{*} \frac{\partial f}{\partial t}-f \frac{\partial f^{*}}{\partial t}\right)-\frac{1}{2}\left|\frac{\partial f}{\partial z}\right|^{2}-\frac{1}{2}\left(\frac{1}{\sigma^{2}}+\sigma^{2}\right)|f|^{2}-\frac{1}{2} g(z) \frac{|f|^{4}}{\sigma^{2}} .
$$

Varying it with respect to $f^{*}(z, t)$ and $\sigma(z, t)$ gives rise to the system of Euler-Lagrange equations:

$$
\begin{aligned}
i \frac{\partial f}{\partial t} & =\left[-\frac{1}{2} \frac{\partial^{2}}{\partial z^{2}}+\frac{1}{2}\left(\frac{1}{\sigma^{2}}+\sigma^{2}\right)+g(z) \frac{|f|^{2}}{\sigma^{2}}\right] f, \\
\sigma^{4} & =1+g(z)|f|^{2},
\end{aligned}
$$

Inserting Eq. (23) into Eq. (22), we obtain a closed-form equation for the axial wave function, which is tantamount to the NPSE derived in Ref. [21], but with the $z$-dependent interaction strength, $g(z)$ :

$$
i \frac{\partial f}{\partial t}=\left[-\frac{1}{2} \frac{\partial^{2}}{\partial z^{2}}+\frac{1+(3 / 2) g(z)|f|^{2}}{\sqrt{1+g(z)|f|^{2}}}\right] f .
$$


In the weak-coupling regime, i.e., $|g(z)||f(z, t)|^{2} \ll 1$, one can expand the nonpolynomial term in Eq. (24), arriving at the cubic-quintic nonlinear Schrödinger (with term 1 representing here the transverse ground-state energy),

$$
i \frac{\partial f}{\partial t}=\left[-\frac{1}{2} \frac{\partial^{2}}{\partial z^{2}}+1+g(z)|f|^{2}+\frac{3}{8} g(z)^{2}|f|^{4}\right] f .
$$

The cubic-quintic nonlinearity for the tightly confined BEC was also derived by means of different approaches [29]. On the other hand, in the strong-coupling regime, $g(z)|f(z, t)|^{2} \gg 1$ (which is relevant only for the repulsive sign of the nonlinearity, $g>0$ ), the NPSE amounts to the nonlinear Schrödinger equation with the quadratic nonlinearity (see, e.g., Ref. [32]):

$$
i \frac{\partial f}{\partial t}=\left[-\frac{1}{2} \frac{\partial^{2}}{\partial z^{2}}+\frac{3}{2} \sqrt{g(z)}|f|\right] f .
$$

Here we aim to analyze bright solitons within the framework of Eq. (24) with $g(z)$ given by Eq. (44), with $g_{0}=0$ and $g_{1}<0$, as said above. Results were obtained from numerical solutions based the finite-difference Crank-Nicolson predictor-corrector algorithm [33].

First, by simulating the NPSE in imaginary time, we study the formation of bright solitons. In particular, at $g_{1}=-0.4$, the soliton does not self-trap, slowly degenerating towards a uniform configuration along axial direction $z$. Instead, at $g_{1}=-1$ the bright soliton self-traps quickly, representing the ground state of Eq. (24). In Fig. 3 we plot typical examples of the axial density, $\rho(z)$, of the so obtained stable bright soliton trapped in the NL, comparing the NPSE results to those predicted by Gaussian ansatz (6) (the stability of the solitons was verified by real-time simulations, see below). The figure shows that the stable solitons are localized around one potential minimum of the NL (at $x=0)$. The NPSE profiles are quite close to their variational counterparts, see also the left panels in Fig. 1. The main quantitative, although not very large, difference between the VA and NPSE is the prediction of the critical strength for the onset of the collapse, as seen in Fig. 2.

\section{Real-time dynamics}

\subsection{Stability of the solitons}

The next step is the study of the real-time dynamics of the quasi-1D BEC trapped in the NL. First of all, simulating NPSE (24) in real time, we have found that all the existing solitons are stable, with initially perturbed wave functions featuring small oscillations around the solitonic configurations. As shown in Fig. 4, an interesting dynamical feature is observed if the initial wave function is the Gaussian with a width close to that of the soliton: expulsion of two small waves from the Gaussian peak (which is represented by the column centered at $z=0$ in Fig. 4). The emitted waves rapidly move in opposite directions, while the remaining central peak relaxes into a stationary soliton. This effect is interesting because the same is not observed in linear lattices, which would readily 


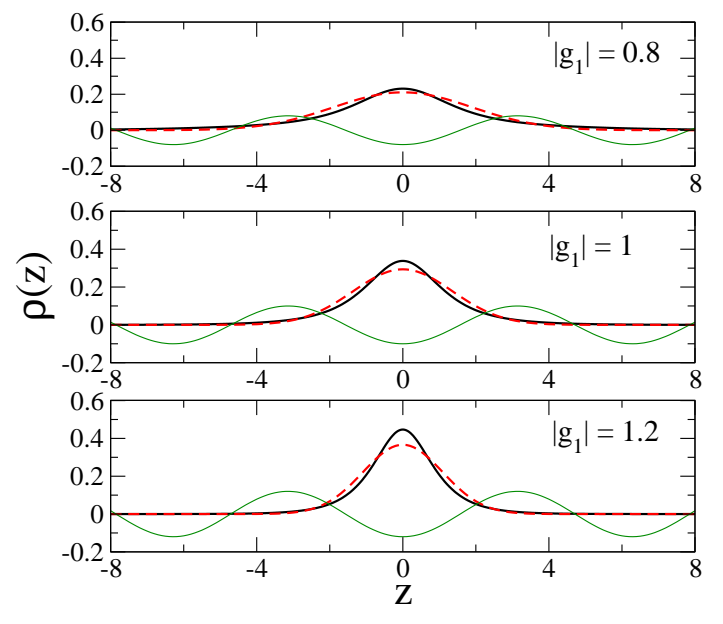

Figure 3. (Color online) Typical examples of axial density $\rho(z) \equiv|f(z)|$ of stable solitons. The solid and dashed lines display the results produced by the NPSE and variational approximation based on ansatz (6), respectively, for three different values of the interaction strength $\left|g_{1}\right|$, fixing $g_{0}=0$ and $k=0.5$. Here and in Figs. 4, 5 and 6. the green sinusoidal line represents the periodic modulation function of the local nonlinearity defined in Eq. (4).

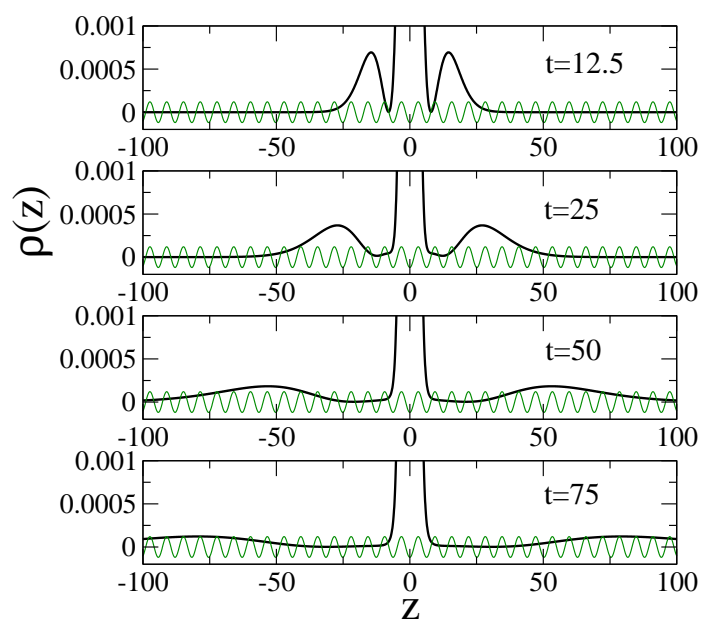

Figure 4. (Color online) Dynamics of a Gaussian wave packet with the initial shape close to the ground-state soliton. The axial density, $\rho(z)$, is displayed at different values of real time $t$, as obtained from simulations of Eq. (24). At $t=0$ (the initial condition, not shown here), there is only the Gaussian centered at $z=0$, with axial width 1.7. The parameters are $g_{0}=0, g_{1}=-1.2$, and $k=0.5$.

trap the radiation "garbage" emitted by the central peak, while the NL is not felt by the small-amplitude waves, hence they may escape freely.

\subsection{Immobility of the trapped solitons}

The mobility of solitons trapped in the NL can be tested by applying a kick to initially quiescent solitons [8]. For this purpose, Eq. (24) was simulated with initial 


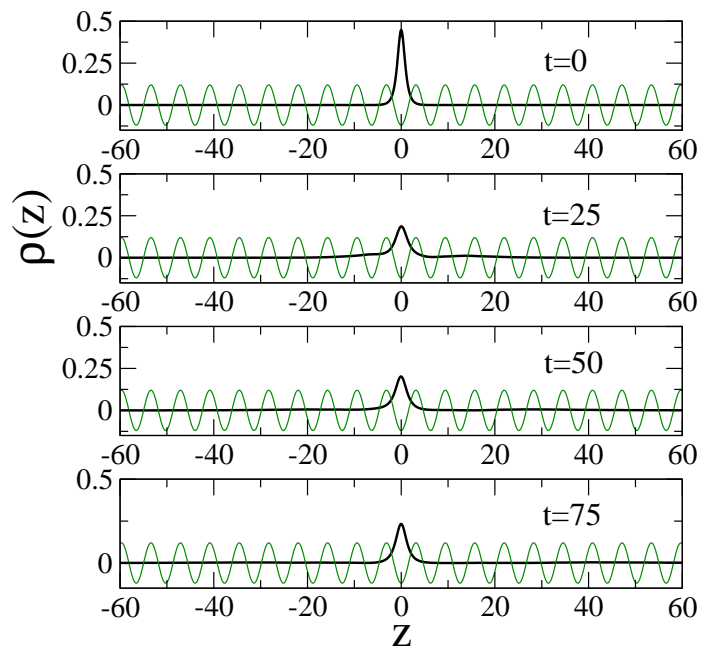

Figure 5. (Color online) The evolution of the kicked soliton with initial velocity $v=0.4$. Axial density $\rho(z)$ is plotted at different values of real time $t$, as obtained from simulations of Eq. (24). Here, $g_{0}=0, g_{1}=-1.2$, and $k=0.5$ are fixed.

condition $f(z, t=0)=f_{\text {sol }}(z) e^{i v z}$, where $v$ is the magnitude of kick, i.e., the initial velocity imparted to soliton $f_{\text {sol }}(z)$, which was produced by means of the imaginary-time simulations of the same NPSE. To present a typical result, we fix $g_{0}=0, g_{1}=-1.2$, and $k=0.5$, and perform real-time simulations at increasing values of $v$. As shown in Fig. 5, at $v=0.4$ we observe ejection of small-amplitude waves from the soliton (cf. Fig. (4), while the central peak remains trapped at the initial position, relaxing back into a stationary soliton, with a somewhat smaller value of the norm.

With the increase of $v$, the amplitude and the velocity of the ejected waves increases, but the remaining soliton stays put. This is a noteworthy difference from the soliton dynamics in 1D NLs with the cubic nonlinearity, where the soliton may be set in motion by the kick [8]. Eventually, if the kick is too strong, it destroys the soliton. In particular, for $\left|g_{1}\right|=1.2$ the destruction is observed at $v \geq 0.48$, see an example in Fig. 6 for $v=0.6$.

The critical velocity, $v_{c}$, at which the kicked soliton is destroyed, is shown in Fig. 7 as a function of the NL strength, $\left|g_{1}\right|$. The figure features a linear growth of $v_{c}$ with NL strength $\left|g_{1}\right|$, at sufficiently large values of $\left|g_{1}\right|$. This fact can be explained by estimating the critical velocity as that at which the respective kinetic energy of the kicked soliton, $(1 / 2) M_{\text {sol }} v^{2}$, is equal to height $V_{\mathrm{PN}}$ of the effective Peierls-Nabarro potential induced by the nonlinear (pseudo) potential. The mass of the soliton, $M_{\text {sol }}$, is proportional to its norm, which is 1, according to Eq. (20). Further, the potential-energy density corresponding to Eq. (24) actually coincides with the potential part of Lagrangian density (21), that should be evaluated with the help of expression (23) for $\sigma$. Then, a straightforward consideration of Eqs. (21), (23) and (24) yields the following scaling relations in the limit of large $\left|g_{1}\right|:|f(0)|^{2} \sim \sigma^{-1} \sim W^{-1} \sim\left|g_{1}\right|^{-1}$, where $W$ is the axial size of the soliton, and, eventually, $V_{\mathrm{PN}} \sim\left|g_{1}\right|^{2}$. Thus, the threshold condition, $(1 / 2) M_{\mathrm{sol}} v^{2}=V_{\mathrm{PN}}$, explains the linear proportionality between $v_{c}$ and $\left|g_{1}\right|$, which is 


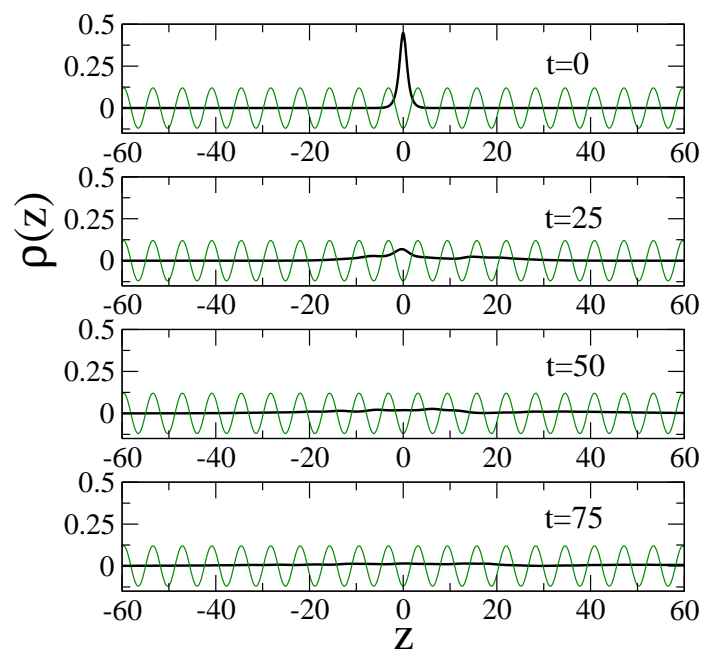

Figure 6. (Color online) The same as in Fig. 5, but for the soliton kicked with initial velocity $v=0.6$.

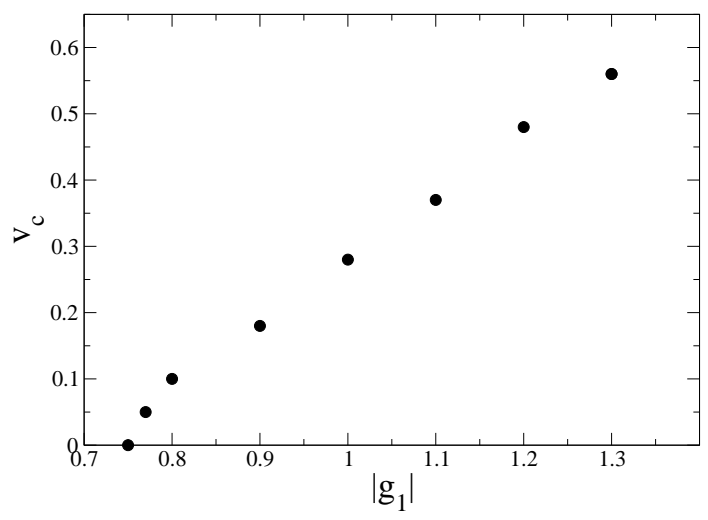

Figure 7. The critical velocity, $v_{c}$, for the destruction of the kicked soliton versus the strength of the nonlinear lattice, $\left|g_{1}\right|$. Here, $g_{0}=0$ and $k=0.5$ are fixed, as before.

observed in Fig. 7 at large $\left|g_{1}\right|$. The vanishing of $v_{c}$ at $\left|g_{1}\right| \approx 0.75$ in Fig. 7 is a consequence of the fact that the soliton with this value of $\left|g_{1}\right|$ lies at the edge of the triangular area in Fig. 2, i.e., it does not exist as a stable mode even without being kicked.

The fact that the kick induces emission of radiation from the soliton may be used to stabilize them in the collapse domain of Fig. 2. To this end, we take, for example, the Gaussian wave packet with $g_{1}=-1.5$ and $k=0.5$, which falls into the region of the collapse. Real-time simulations of Eq. (24) demonstrate that, if the Gaussian is kicked hard enough, it does not blow up, but rather forms a stable soliton, in a combination with the emission of radiation waves. This is shown in Fig. 8 for initial velocity $v=0.4$. The initial configuration evades the blowup because the emission of radiation reduces the norm of the remaining soliton, pushing it beneath the collapse threshold, see the lower panel in Fig. 8. This observation suggests that the relaxation of the perturbed 

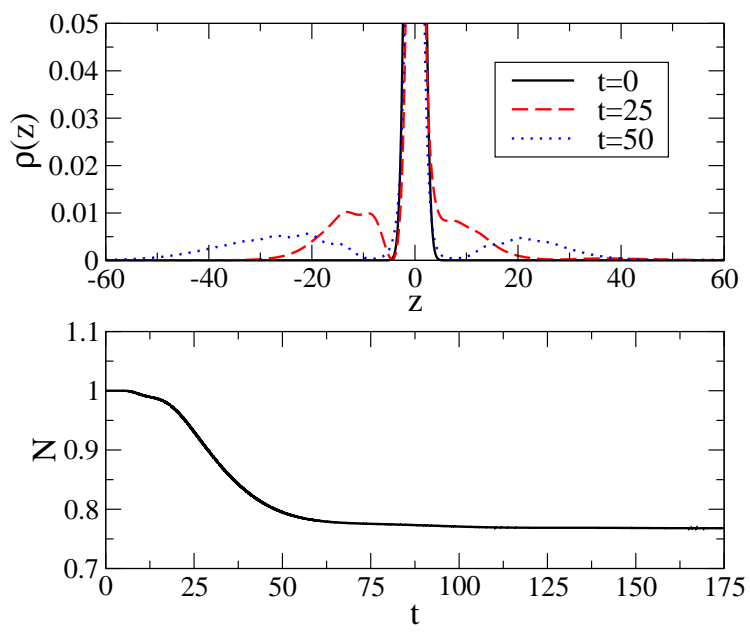

Figure 8. (Color online) Upper panel: The dynamics of the Gaussian wave packet, kicked with initial velocity $v=0.4$, whose parameters belong to the collapse domain in terms of Fig. 2. $g_{1}=-1.5, k=0.5$, and $g_{0}=0$. At $t=0$ (the initial condition shown by the solid line), there is only the column centered at $z=0$, which represents the Gaussian of axial width 1.7. Lower panel: the norm of the wave function in interval $-15<x<15$, as a function of time.

soliton via the emission of radiation proceeds faster than the onset of the collapse, which attests to the robustness of the solitons. Finally, as expected, if the kick is too hard (in the present case, this means $v \geq 0.6$ ), it destroys the Gaussian wave packet, causing its complete decay into radiation.

\section{Conclusions}

We have reported results for 3D matter-wave solitons supported by a combination of the axial 1D NL (nonlinear lattice), which periodically reverses the sign of the nonlinear interaction, and the tightly trapping harmonic-oscillator potential acting in the transverse plane. The results were obtained by means of two distinct approaches: The VA (variational approximation), which was applied directly to the 3D GrossPitaevskii equation, and the 1D NPSE, that was derived from the underlying 3D equation. Previous works did not study the stabilization of $3 \mathrm{D}$ solitons by NLs. The main result, produced by means of both methods in similar forms, is the stability domain for solitons in the plane of the NL strength and wavenumber. The usual 1D cubic GrossPitaevskii equation with the NL cannot produce adequate results, as it does not give rise to the collapse, which is the most important stability-limiting factor.

Another essential difference of the solitons produced by the NPSE with the NL from their counterparts in the case of the cubic NL is that the solitons are immobile in the framework of the NPSE: The kick applied to the soliton either leaves it pinned, or, eventually, destroys it. The critical size of the kick which destroys the soliton was found to be proportional to the strength of the NL, provided that the strength is large 
enough; an explanation for this dependence was proposed. On the other hand, the kick, if applied to the wave packet created above the collapse threshold, may help it to shed off the excess norm and thus stabilize itself against the collapse. A related dynamical effect, which demonstrates the difference of the NLs from linear lattices, is that wave

packets relaxing into solitons can emit small-amplitude waves, which freely propagate in the system.

A challenging extension of the analysis may be to develop it for the setting with a 2D NL and the 1D trapping potential acting in the transverse direction. The 2D version of the NPSE was developed previously, but in the absence of the NL [21, 34]. In this case, one may expect the existence of both fundamental and vortical 2D solitons.

\section{References}

[1] V. A. Brazhnyi, and V. V. Konotop, Mod. Phys. Lett. B 18, 627 (2004); O. Morsch and M. Oberthaler, Rev. Mod. Phys. 78, 196 (2006).

[2] B. A. Malomed, D. Mihalache, F. Wise, and L. Torner, J. Optics B: Quant. Semicl. Opt. 7, R53 (2005).

[3] Y. V. Kartashov, V. A. Vysloukh, and L. Torner, Progress in Optics 52, 63 (ed. by E. Wolf: North Holland, Amsterdam, 2009).

[4] Y. V. Kartashov, B. A. Malomed, and L. Torner, Rev. Mod. Phys. 83, 247 (2011).

[5] B. B. Baizakov, B. A. Malomed, and M. Salerno, Europhys. Lett. 63, 642 (2003); Phys. Rev. A 70, 053613 (2004); J. Yang and Z. H. Musslimani, Opt. Lett. 28, 2094 (2003).

[6] T. Mayteevarunyoo, B. A. Malomed, and G. Dong, Phys. Rev. A 78, 053601 (2008).

[7] L. Salasnich, A. Cetoli, B. A. Malomed, F. Toigo, and L. Reatto, Phys. Rev. A 76, 013623 (2007).

[8] H. Sakaguchi and B. A. Malomed, Phys. Rev. E 72, 046610 (2005); Phys. Rev. A 81, 013624 (2010).

[9] P. Niarchou, G. Theocharis, P. G. Kevrekidis, P. Schmelcher, and D. J. Frantzeskakis, Phys. Rev. A 76, 023615 (2007); L. Zhou, C. Xue, Y. Qi, and S. Lou, Phys. Lett. A 372, 4395 (2008).

[10] F. K. Abdullaev and J. Garnier, Phys. Rev. A 72, 061605(R) (2005); F. Abdullaev, A. Abdumalikov, and R. Galimzyanov, Phys. Lett. A 367, 149 (2007); F. K. Abdullaev, A. Gammal, M. Salerno, and L. Tomio, Phys. Rev. A 77, 023615 (2008).

[11] H. L. F. da Luz, F. K. Abdullaev, A. Gammal, M. Salerno, and L. Tomio, Phys. Rev. A 82, 043618 (2010).

[12] G. Theocharis, P. Schmelcher, P. G. Kevrekidis, and D. J. Frantzeskakis, Phys. Rev. A 72, 033614 (2005); G. Theocharis, P. Schmelcher, P. G. Kevrekidis, and D. J. Frantzeskakis, ibid. 74, 053614 (2006); A. S. Rodrigues, P. G. Kevrekidis, M. A. Porter, D. J. Frantzeskakis, P. Schmelcher, and A. R. Bishop, ibid. 78, 013611 (2008).

[13] Y. Sivan, G. Fibich, and M. I. Weinstein, Phys. Rev. Lett. 97, 193902 (2006).

[14] Y. Kominis and K. Hizanidis, Opt. Express 16, 12124 (2008); K. Hizanidis, Y. Kominis, and N. K. Efremidis, ibid. 16, 18296 (2008).

[15] J. Belmonte-Beitia, V. M. Pérez-García, V. Vekslerchik, and P. J. Torres, Phys. Rev. Lett. 98, 064102 (2007); J. Belmonte-Beitia, V. V. Konotop, V. M. Pérez-García, and V. E. Vekslerchik, Chaos Solitons Fractals 41, 1158 (2009); V. Perez-Garcia and R. Pardo, Physica D 238, 1352 (2009).

[16] L.-C. Qian, M. L. Wall, S. Zhang, Z. Zhou, and H. Pu, Phys. Rev. A 77, 013611 (2008); Z.-W. Zhou, S.-L. Zhang, X.-F. Zhou, G.-C. Guo, X. Zhou, and H. Pu, ibid. 83, 043626 (2011).

[17] C. H. Tsang, B. A. Malomed, and K. W. Chow, Discrete Contin. Dyn. Syst. Ser. S 4, 1299 (2011).

[18] H. Sakaguchi and B. A. Malomed, Phys. Rev. E 73, 026601 (2006); Y. Sivan, G. Fibich, and M. I. Weinstein, Phys. Rev. Lett. 97, 193902 (2006); Y. V. Kartashov, B. A. Malomed, V. A. 
Vysloukh, and L. Torner, Opt. Lett. 34, 770 (2009); Y. Sivan, G. Fibich, B. Ilan, and M. I. Weinstein, Phys. Rev. E 78, 046602 (2008); N. V. Hung, P. Ziń, M. Trippenbach, and B. A. Malomed, ibid. 82, 046602 (2010); T. Mayteevarunyoo, B. A. Malomed, and A. Reoksabutr, J. Mod. Opt., in press (DOI: 10.1080/09500340.2011.601329).

[19] M. Matuszewski, E. Infeld, B. A. Malomed, and M. Trippenbach, Phys. Rev. Lett. 95, 050403 (2005).

[20] A. Muñoz Mateo, V. Delgado, and B. A. Malomed, Phys. Rev. A 82, 053606 (2010); ibid. 83, 053610 (2011).

[21] L. Salasnich, Laser Phys. 12, 198 (2002); L. Salasnich, A. Parola, and L. Reatto, Phys. Rev. A 65, 043614 (2002); ibid. 66, 043603 (2002); L. Salasnich and B. A. Malomed, ibid. Phys. Rev. A 74, 053610 (2006).

[22] A. Muñoz Mateo and V. Delgado, Phys. Rev. A 75, 063610 (2007); 77, 013617 (2008); Ann. Phys. (N.Y.) 324, 709 (2009).

[23] L. Salasnich, A. Cetoli, B. A. Malomed, and F. Toigo, Phys. Rev. A 75, 033622 (2007).

[24] M. Theis, G. Thalhammer, K. Winkler, M. Hellwig, G. Ruff, R. Grimm, and J. H. Denschlag, Phys. Rev. Lett. 93, 123001 (2004).

[25] S. Inouye, M. R. Andrews, J. Stenger, H. J. Miesner, D.M. Stamper-Kurn, and W. Ketterle, Nature (London) 392, 151 (1998).

[26] S. Ghanbari, T. D. Kieu, A. Sidorov, and P. Hannaford, J. Phys. B: At. Mol. Opt. Phys. 39, 847 (2006).

[27] K. E. Strecker, G. B. Partridge, A. G. Truscott, and R. G. Hulet, New J. Phys. 5, 73.1 (2003).

[28] F. Kh. Abdullaev and M. Salerno, Phys. Rev. A 72, 033617 (2005); G. L. Alfimov, V. V. Konotop, and P. Pacciani, Phys. Rev. A 75, 023624 (2007).

[29] A. E. Muryshev, G. V. Shlyapnikov, W. Ertmer, K. Sengstock, and M. Lewenstein, Phys. Rev. Lett. 89, 110401 (2002); L. D. Carr and J. Brand, Phys. Rev. Lett. 92, 040401 (2004); Phys. Rev. A 70, 033607 (2004); L. Khaykovich and B.A. Malomed, Phys. Rev. A 74, 023607 (2006).

[30] B. B. Baizakov and M. Salerno, Phys. Rev. A 69, 013602 (2004).

[31] L. Salasnich, Int. J. Mod. Phys. B 14, 1 (2000).

[32] J. Fujioka, E. Cortés, R. Pérez-Pascual, R. F. Rodríguez, A. Espinosa, and B. A. Malomed, Chaos 21, 033120 (2011).

[33] E. Cerboneschi, R. Mannella, E. Arimondo, and L. Salasnich, Phys. Lett. A 249, 495 (1998); G. Mazzarella and L. Salasnich, Phys. Lett. A 373, 4434 (2009).

[34] L. Salasnich and B. A. Malomed, Phys. Rev. A 79, 053620 (2009); L. Salasnich and B. A. Malomed, Spontaneous symmetry breaking in linearly coupled disk-shaped Bose-Einstein condensates, Molecular Physics, in press (DOI:10.1080/00268976.2011.602370). 